\title{
Are Migraine and Tension-Type Headache Genetically Related? An Investigation of Twin Family Data
}

\author{
Lannie Ligthart, Annemarie Huijgen, Gonneke Willemsen, Eco J. C. de Geus, and Dorret I. Boomsma \\ Department of Biological Psychology, Netherlands Twin Register, Vrije Universiteit, Amsterdam, The Netherlands
}

\begin{abstract}
Migraine and tension-type headache (TTH) are often viewed as distinct entities and defined as such in the International Classification of Headache Disorders, 2nd edition (ICHD-II) criteria, although there is also empirical evidence to suggest they may be etiologically similar. This study aims to investigate whether migraine and TTH are etiologically related conditions. First, we explored whether migraine and TTH were associated with the same environmental and lifestyle risk factors at the population level. Second, we examined comorbidity of migraine and TTH in a twin design. By comparing the associations in monozygotic (MZ) and dizygotic (DZ) twin pairs, we investigated whether the comorbidity can be explained by genetic factors that influence both conditions. Results indicated that migraine and TTH were largely associated with the same environmental and lifestyle factors, including younger age, female sex, higher body mass index, more depression, stress at home, and less participation in regular exercise, with consistently stronger effects for migraine than for TTH. Migraine in one twin was significantly associated with TTH in the other twin. A stronger cross-trait, cross-twin association in MZ than DZ twins suggested that this comorbidity may also be partly due to shared genetic factors, although the difference in associations was not significant. In conclusion, our findings are consistent with the hypothesis that migraine and TTH have partly shared etiologies. For both treatment and research, it may be advisable not to make a rigid distinction, but to treat migraine and TTH as related conditions.
\end{abstract}

Keywords: migraine, tension-type headache, shared environmental risk, discordant twin design

The most common primary headaches seen in medical practice are migraine and tension-type headache (TTH; Rasmussen et al., 1991). The International Classification of Headache Disorders (ICHD) criteria have always classified these two conditions as distinct entities that are mutually exclusive in terms of symptoms: essentially, the diagnostic criteria for TTH are the negation of those for migraine; for example, not bilateral, non-pulsating, and not aggravated by physical activity (Headache Classification Committee of the International Headache Society, 1988, 2004, 2013). However, a migraine attack may include symptoms of TTH and vice versa, and many patients experience attacks of both types (Kaniecki, 2002), suggesting a fair amount of comorbidity between the two conditions. This raises the question whether migraine and TTH are distinct disorders, or whether they occupy the severe and mild end of a severity continuum, with TTH lying on the mild end and migraine lying on the severe end of the spectrum (the continuumseverity theory; Waters, 1973).

Several studies support the distinction between migraine and TTH. For example, a Danish population study by
Ulrich et al. (1996) reported that the 1-year prevalence and sex ratio of TTH did not differ in individuals with and without migraine, suggesting they are independent conditions. Another Danish study by the same group emphasized the phenotypic differences between migraine and TTH, reporting that sex ratio and age at onset differed between them (Rasmussen, 1996). In a review published in 2010, Blumenfeld et al. argued that TTH is distinct from migraine in its pathophysiology and clinical presentation, and while mild migraines resemble TTH attacks, these attacks can be effectively treated with triptans, whereas pure TTH patients do not respond well to triptan therapy.

In contrast, a considerable number of studies support the view of migraine and TTH being manifestations of the

RECEIVED 27 January 2018; ACCEPTED 30 January 2018

ADDRESS FOR CORRESPONDENCE: Dr. Lannie Ligthart, Department of Biological Psychology, Netherlands Twin Register, Vrije Universiteit Amsterdam, van der Boechorststraat 1, 1081 BT Amsterdam, The Netherlands. E-mail: rsl.ligthart@vu.nl 
same disorder, based on the frequent co-occurrence of TTH and migraine in the same patients, similarities in epidemiological features, and precipitating factors common to both conditions (Drummond \& Lance, 1984; Featherstone, 1985; Kaniecki, 2002; Leston, 1996; Russell et al., 2006; Spierings et al., 2001; Vargas, 2008). However, migraine and TTH are still treated as distinct entities in the latest edition of the ICHD (version III-beta; Headache Classification Committee of the International Headache Society, 2013).

Whether or not migraine and TTH are distinct entities has important implications, both from a clinical perspective and in research settings. In clinical practice, the choice of treatment is likely to depend on the diagnosis, in which case misdiagnosis of migraine as TTH (or vice versa) may result in inadequate treatment (Kaniecki, 2002). In research, treating migraine and TTH as separate entities versus treating them as one condition can have a great impact on study outcomes. For example, in recent years, several large genomewide association (GWA) studies have been conducted for migraine, with the aim of identifying the genes underlying this condition (Anttila et al., 2013; Chasman et al., 2011; Freilinger et al., 2012; Gormley et al., 2016). TTH patients are typically excluded from GWA studies, based on the assumption that TTH is genetically different from migraine. However, given the literature described above, it is unclear whether this assumption is correct. Since large sample sizes are essential in gene-finding studies, this question is of importance.

The aim of the present study was twofold. First, we examined the similarities and differences between migraine and TTH with respect to associated environmental and lifestyle factors in a population-based sample of Dutch twins and their families. Previous studies have reported strong similarities in the risk factors associated with migraine and TTH, and typically, the association is stronger for migraine than for TTH. This could be interpreted as evidence for the continuum-severity theory. Factors potentially related to headache and investigated in this study are body mass index (BMI) (Bigal \& Lipton, 2006; Queiroz et al., 2009), current smoking (Scher et al., 2005), anxious depression (Breslau et al., 2003; Ligthart et al., 2013; Merikangas et al., 1993), loneliness (Stensland et al., 2014), exercise (Fricton et al., 2009), diabetes (Aamodt et al., 2007; Hagen et al., 2017), and stress (Haque et al., 2012; Rasmussen, 1993; Schoonman et al., 2007; Spierings et al., 2001). Studying a large number of traits in a large population-based sample should allow us to detect patterns in how these traits are related to migraine and TTH: if the two conditions differ merely in severity on the same spectrum, they are expected to be associated with the same risk factors, with migraine showing stronger effects than TTH. Second, we focused on the twins in the sample to investigate whether migraine and TTH are genetically related conditions. We are not aware of any previous studies addressing this question. Twin data can be used to ex- amine the influence of genetic and environmental influences on individual traits (in this case the occurrence of migraine and TTH), as well as on the overlap between traits (Boomsma et al., 2002). If migraine and TTH are (partly) influenced by the same genetic or shared environmental factors, it was expected that in co-twins of migraineurs we would observe a higher prevalence of TTH than in co-twins of non-migraineurs. In the case of shared underlying genetic factors, we expected this resemblance to be stronger in monozygotic (MZ) than dizygotic (DZ) twins, because $M Z$ twins are genetically identical, whereas DZ twins share only $50 \%$ of their segregating genes on average.

\section{Methods}

\section{Study Population}

The Netherlands Twin Registry (NTR) is a nationwide population-based register where adult twins and their relatives (parents, siblings, spouses, and offspring) can register (Willemsen et al., 2013). Participants are invited every 2-4 years to complete a survey that contains questions about health, lifestyle, and personality. The present study was based on headache data from the ninth wave of data collection (2011-2012) for adult participants (survey 9), which focused on headache, pain, and anxiety. The data collection has been described in detail elsewhere (Ligthart et al., 2014). Data on risk factors associated with migraine and TTH were taken from survey 9 and from the previous survey (survey 8 ), which was conducted 2 years earlier. This was a study with a focus on alcohol use, smoking, and personality (Geels et al., 2013).

A total of 11,947 participants completed survey 9 , which equaled a total response rate of $43 \%$ (Willemsen et al., 2013). Six individuals were excluded because they were younger than 18 years, resulting in a sample of 11,941 individuals. In total, $48 \%$ of the participants in this study were twins. Of the total sample, 10,040 individuals also completed survey 8 .

\section{Measures}

Migraine and tension-type headache. Diagnosis of the headache was determined with questions on headache symptomatology based on the ICHD-II criteria (Headache Classification Committee of the International Headache Society, 2004) in survey 9. Participants were asked to report both current and past headache symptoms to assess the lifetime presence of migraine. Headaches due to cold, fever, and alcohol or drugs were excluded. Since the diagnoses of migraine and TTH are mutually exclusive, an individual can have either a diagnosis of TTH or migraine, but not both. Hence, each individual was classified into one of three categories: unaffected (i.e., no headache), TTH, or migraine. Individuals with headaches not meeting the criteria for TTH or migraine were classified as having 
'undefined headache'. This resulted in 4,951 individuals screening negative for current headache, 1,814 with migraine, 2,770 with TTH, 2,004 with undefined headaches, and 402 who could not be classified due to insufficient data. For lifetime headaches, 3,930 individuals screened negative, 2,159 were classified as migraineurs, 3,053 had TTH, 2,360 had undefined headache, and 439 had insufficient data. Individuals with undefined headache or insufficient data were excluded from the analyses.

The analyses addressing comorbidity and genetic overlap included lifetime diagnoses, which best reflect the individual's overall risk of the disorder. For the analysis of health and lifestyle factors related to TTH and migraine, current headache status was analyzed, to capture the associations between these factors and headache at the time of measurement.

Environmental and lifestyle variables. To investigate the role of demographics, environment and lifestyle in the development of migraine and $\mathrm{TTH}$, the following variables were included in the analysis: age, sex, BMI, smoking and anxious depression, regular exercise behavior (from survey 9 ), and diabetes, financial stress, stress at work, stress at home, and loneliness (from survey 8 ).

BMI (kg/height in $\mathrm{m}^{2}$ ) was measured by asking the participants to report their height and weight.

Current smoking was assessed by the question 'Do you currently smoke?' (yes or no).

Anxious depression was measured using the anxious depression subscale of the Adult Self Report (Achenbach \& Rescorla, 2003). This is a continuous measure that reflects a combination of anxiety and depressive symptoms and consists of the sum of 18 items rated on a 3 -point scale $(0=$ not true, $1=$ somewhat or sometimes true, $3=$ very true or often true).

Diabetes: Participants were asked if they had ever been diagnosed with diabetes by a physician. Possible answers were 'no', 'yes, in the past', and 'yes, currently'. Stress: Three types of stress were measured: financial stress (What level of financial stress have you experienced in the past year?), stress at work (How often have you felt stress at work in the past year?), and stress at home (How often did you experience stress at home in the past year?).

Regular exercise behavior was measured in weekly 'metabolic equivalent of task' (MET) hours. Regular voluntary exercise activities (excluding non-leisure physical activity such as cycling to work and household work) were assessed with items addressing the type of exercise, the number of years, times per week, and average duration. Information on the type of exercise was recoded into a weekly MET using Ainsworth's compendium of physical activities (Ainsworth et al., 2000). Weekly MET hours were calculated by multiplying the MET score by the number of hours per week for each exercise activity, summed across all exercise activities reported.
Loneliness was assessed using the Three Item Loneliness Scale (TILS; Hughes et al., 2004). The items included in this scale are 'How often do you feel left out?', 'How often do you feel isolated from others?', and 'How often do you feel that you lack companionship?' The items have three response categories: $1=$ almost never, $2=$ sometimes, and $3=$ often, and are summed.

\section{Statistical Analysis}

Association of migraine and TTH with environmental and lifestyle variables. Multiple logistic regression analysis was performed to test the association of current migraine and TTH with environmental and lifestyle factors. Three analyses were performed. The first two analyses tested whether risk factors were predictive of migraine and TTH. To investigate the similarities and differences between migraine and TTH, a third analysis was done in which migraine patients were compared to TTH patients to see whether the two conditions were differently associated with the variables of interest. Participants with other headaches or unknown headache status were excluded. The regression analyses were performed in Stata 13 (StataCorp LP, College Station, TX). A robust variance estimator was used, and family ID was included as a cluster variable to adjust standard errors for the non-independence of observations within a family. Significance tests were performed at an alpha level of .05.

Comorbidity of migraine and TTH within twin pairs. To investigate the comorbidity between migraine and TTH, we analyzed data on lifetime prevalence of migraine and TTH from twin pairs. First, we assessed the prevalence of migraine in the co-twins of migraineurs, and the prevalence of TTH in the co-twins of individuals with TTH. This was done for all same-sex twin pairs and for $\mathrm{MZ}$ and $\mathrm{DZ}$ twins separately. Given that both migraine and TTH are partly genetic conditions (Mulder et al., 2003; Russell et al., 2007), it is expected that a patient's co-twin will be at increased risk of the condition, compared to a non-patient's co-twin, and that the risk is more strongly increased in MZ than DZ cotwins. This is because MZ twins are genetically identical, whereas DZ twins share only half of their segregating genes, on average. Next, we tested whether individuals whose cotwin had migraine were at increased risk of TTH compared to individuals whose co-twin did not have migraine. Again, this was done for all twin pairs and for $\mathrm{MZ}$ and $\mathrm{DZ}$ twins separately. If the risk is more strongly increased in MZ than DZ co-twins, this indicates that the comorbidity is at least partly explained by genetic factors shared between the two conditions. To assess the difference in risk between co-twins of cases versus non-cases, sex-adjusted odds ratios were calculated, with $95 \%$ confidence intervals to assess the significance of the effect. This was done with the logistic regression procedure in SPSS 20 (SPSS Inc., Chicago, IL, USA). 


\begin{tabular}{|c|c|c|}
\hline \multicolumn{3}{|c|}{ Demographic Characteristics of Total Sample Versus Twins Only } \\
\hline Variables & Total sample, $N( \pm S D$ or \%) & Twins only, $N( \pm S D$ or \%) \\
\hline Sample size & 11,941 & 5,854 \\
\hline Mean age & $44.05( \pm 15.7)$ & $36.2( \pm 14.5)$ \\
\hline Females (\%) & $7,366(61.7 \%)$ & $3,974(67.9 \%)$ \\
\hline Migraine (current) & $1,818(15.2 \%)$ & 934 (16.0\%) \\
\hline Migraine (lifetime) & $2,159(18.1 \%)$ & $1,047(17.9 \%)$ \\
\hline Tension-type headache (current) & $2,791(23.4 \%)$ & $1,440(24.6 \%)$ \\
\hline Tension-type headache (lifetime) & $3,081(25.8 \%)$ & $1,565(26.7 \%)$ \\
\hline
\end{tabular}

\section{Results}

Table 1 shows the demographic characteristics of the study population for the total sample and the twins (48\% of the total sample). There were no significant differences in these characteristics between the total sample and the twin sample. In the entire cohort, we first investigated the relationship between migraine, TTH, and environmental and lifestyle traits. Table 2 shows the results of a multiple regression analysis with migraine and TTH as the dependent variables, and the environmental and lifestyle variables as the independent variables. Migraine was positively associated with younger age, female sex, higher BMI, higher anxious depression scores, occasional, regular and constant stress at home, and loneliness. The presence of diabetes and a high regular exercise score, on the other hand, was associated with a lower risk of migraine.

TTH was associated largely with the same risk factors; however, no significant association with diabetes, loneliness, and regular and constant stress at home was observed. The only factor associated with TTH but not migraine was smoking, which showed a negative association with TTH. For migraine, an odds ratio in the same direction was observed, but this association was not significant.

In general, effect sizes were larger for migraine than TTH: these differences were significant for sex $(p<.001)$, BMI $(p=.001)$, anxious depression $(p=.004)$, and stress at home $(p=.007$ and $p<.001$ for regular and constant stress, respectively), indicating that these factors are more strongly associated with migraine than with TTH.

Second, we assessed the extent of comorbidity between current migraine and TTH. We identified 399 twin pairs where both twins had migraine data, 589 pairs where both twins had TTH data, and 430 pairs where one twin had data for migraine and the other for TTH. Table 3 gives the results of these analyses for the total sample and for MZ and DZ twin pairs separately. The first analysis showed that individuals whose co-twin had migraine had a strongly increased risk of migraine themselves $(O R=8.48)$. The increase in risk was particularly strong in $\mathrm{MZ}$ twins compared to $\mathrm{DZ}$ twins with odds ratios of 16.67 and 2.51, respectively, reflecting a clear role of genetic factors in the etiology of migraine. The second analysis compared the risk of TTH in co-twins of individuals with and without TTH, and showed a somewhat similar but less pronounced pattern. The risk of
TTH was significantly increased in individuals whose cotwin had TTH $(O R=2.26)$, but this increase in risk was only slightly stronger for MZ $(O R=2.38)$ than DZ (OR $=2.00$ ) twins. The difference was not significant, as indicated by the $95 \%$ confidence intervals. This suggests that genetic factors are less important in TTH than in migraine. The third analysis (based on 430 twin pairs) compared the risk of migraine in co-twins of individuals with and without TTH. Again, we observed a significantly increased risk, and the increase in risk appeared stronger in $\mathrm{MZ}(O R=2.44)$ than in DZ twins $(O R=1.34)$, although the difference was not significant.

\section{Discussion}

Several interesting observations emerged from this study. First, both migraine and TTH showed a familial pattern, where co-twins of affected individuals had an increased risk of also having the condition. For migraine, this effect was markedly stronger in MZ than DZ twins, confirming that genetic factors play an important role in this condition. In TTH, the effect was only slightly stronger in the MZ twins, suggesting genetic factors play a less prominent role in TTH, at least in the subgroup of twins where neither co-twin has migraine. Furthermore, we observed that migraine in one twin was significantly associated with TTH in the other twin, indicating that a shared etiology is plausible. The effect was somewhat (albeit not significantly) stronger in $\mathrm{MZ}$ than DZ twins, suggesting the comorbidity of migraine and TTH may in part be explained by to genetic factors shared between the two conditions.

The finding that genetic factors were relatively unimportant in TTH is somewhat unexpected. This does not appear to be explained by the headaches being less severe in this group of participants: the twins with TTH included in the analyses did not have less severe or less frequent headaches than the TTH twins not included because their co-twin either had migraine or missing data (results not shown). Previous studies reported moderate heritability for TTH, although results were not entirely consistent. For example, Russell et al. (2007) reported heritability estimates of $48 \%$ in men and $44 \%$ in women. This study differed from ours in that infrequent and frequent episodic or chronic TTH were treated as separate categories. Interestingly, an earlier study 
TABLE 2

Environmental and Lifestyle Variables Associated With Migraine and TTH

\begin{tabular}{|c|c|c|c|c|c|c|}
\hline \multirow[b]{2}{*}{ Risk factor } & \multicolumn{2}{|c|}{ Migraine (versus no headache) } & \multirow[b]{2}{*}{$p$} & \multicolumn{3}{|c|}{ TTH (versus no headache) } \\
\hline & OR $(95 \% \mathrm{Cl})$ & $\mathrm{B}(95 \% \mathrm{Cl})$ & & OR $(95 \% \mathrm{Cl})$ & $\mathrm{B}(95 \% \mathrm{Cl})$ & $p$ \\
\hline Age & $0.98[0.98,0.99]$ & $-0.02[-0.03,0.01]$ & $<.001$ & $0.98[0.98,0.99]$ & $-0.02[-0.02,0.01]$ & $<.001$ \\
\hline Sex & $5.52[4.55,6.70]$ & $1.71[1.52,1.90]$ & $<.001$ & $2.13[1.87,2.41]$ & $0.75[0.63,0.88]$ & $<.001$ \\
\hline BMI & $1.05[1.03,1.07]$ & $0.05[0.03,0.07]$ & $<.001$ & $1.02[1.01,1.04]$ & $0.02[0.01,0.04]$ & .008 \\
\hline Smoking & $0.93[0.74,1.17]$ & $-0.07[-0.30,0.16]$ & .558 & $0.80[0.66,0.97]$ & $-0.22[-0.42,0.03]$ & .021 \\
\hline Anxious depression & $1.06[1.04,1.08]$ & $0.05[0.04,0.07]$ & $<.001$ & $1.04[1.02,1.05]$ & $0.04[0.02,0.05]$ & $<.001$ \\
\hline Diabetes & $0.42[0.21,0.83]$ & $-0.88[-1.57,0.19]$ & .013 & $0.75[0.50,1.14]$ & $-0.29[-0.70,0.13]$ & .180 \\
\hline Financial stress-moderate* & $1.20[0.95,1.50]$ & $0.18[-0.05,0.41]$ & .125 & $0.91[0.76,1.11]$ & $-0.09[-0.28,0.10]$ & .355 \\
\hline Financial stress—high* & $1.33[0.90,1.97]$ & $0.29[-0.11,0.68]$ & .152 & $0.95[0.66,1.35]$ & $-0.06[-0.41,0.30]$ & .759 \\
\hline Work stress-occasionally** & $1.10[0.90,1.35]$ & $0.10[-0.11,0.30]$ & .360 & $1.07[0.91,1.27]$ & $0.07[-0.10,0.24]$ & .417 \\
\hline Work stress—regularly** & $1.14[0.86,1.52]$ & $0.13[-0.16,0.42]$ & .372 & $1.26[1.00,1.59]$ & $0.23[0.00,0.46]$ & .054 \\
\hline Work stress-constantly** & $1.12[0.55,2.27]$ & $0.11[-0.60,0.82]$ & .758 & $1.49[0.87,2.56]$ & $0.40[-0.14,0.94]$ & .148 \\
\hline Stress at home-occasionally** & $1.53[1.25,1.89]$ & $0.43[0.22,0.63]$ & $<.001$ & $1.20[1.04,1.39]$ & $0.18[0.04,0.33]$ & .014 \\
\hline Stress at home-regularly** & $1.79[1.36,2.34]$ & $0.58[0.31,0.85]$ & $<.001$ & $1.24[1.00,1.55]$ & $0.22[0.00,0.44]$ & .050 \\
\hline Stress at home-constantly** & $2.80[1.65,4.72]$ & $1.03[0.50,1.55]$ & $<.001$ & $0.78[0.44,1.41]$ & $-0.24[-0.83,0.34]$ & .416 \\
\hline Weekly MET score & $0.99[0.99,1.00]$ & $-0.01[-0.01,0.00]$ & .001 & $0.99[0.99,1.00]$ & $-0.01[-0.01,0.00]$ & .001 \\
\hline Loneliness & $1.12[1.04,1.20]$ & $0.11[0.04,0.18]$ & .003 & $1.04[0.98,1.10]$ & $0.04[-0.02,0.10]$ & .200 \\
\hline
\end{tabular}

\begin{tabular}{|c|c|c|c|c|}
\hline \multicolumn{5}{|c|}{$\begin{array}{l}\text { Association Between Current Migraine and } \\
\text { Tension-Type Headache Within Twin Pairs, } \\
\text { Adjusted for Sex }\end{array}$} \\
\hline & $n$ & OR & $\mathrm{Cl}$ & $p$ value \\
\hline \multicolumn{5}{|c|}{ Migraine twin 1 versus migraine twin 2} \\
\hline Total & 399 & 8.48 & $5.03,14.30$ & $<.001$ \\
\hline $\mathrm{MZ}$ & 268 & 16.67 & $8.41,33.07$ & $<.001$ \\
\hline DZ & 131 & 2.51 & $1.05,5.98$ & .038 \\
\hline \multicolumn{5}{|c|}{ TTH twin 1 versus TTH twin 2} \\
\hline Total & 589 & 2.26 & $1.59,3.22$ & $<.001$ \\
\hline MZ & 417 & 2.38 & $1.56,3.62$ & $<.001$ \\
\hline $\mathrm{DZ}$ & 172 & 2.00 & $1.04,3.84$ & .037 \\
\hline \multicolumn{5}{|c|}{ Migraine twin 1 versus TTH twin 2} \\
\hline Total & 430 & 2.01 & $1.23,3.27$ & .005 \\
\hline MZ & 297 & 2.44 & $1.34,4.45$ & .004 \\
\hline $\mathrm{DZ}$ & 133 & 1.34 & $0.56,3.21$ & .511 \\
\hline
\end{tabular}

by the same group suggested that while frequent episodic TTH was heritable, infrequent episodic TTH was not, suggesting the heritability of TTH might depend on headache frequency. This pattern was observed in twin pairs with as well as without comorbid migraine (Russell et al., 2006). A possible complicating factor in all studies addressing this is the risk of mild migraine attacks being misclassified as TTH, in which case TTH might be a mixed bag of 'real' TTH and mild migraines (Blumenfeld et al., 2010). This might obscure potential differences in heritability between the two conditions.

In the analyses of predictors of migraine and TTH, similar patterns were observed for both conditions. Both migraine and TTH were significantly associated with age, sex, BMI, anxious depression, stress at home, and regular exercise behavior, and the direction of the effects was highly consistent across both conditions. In general, the observed effects were stronger and more often significant for migraine than for TTH, consistent with the view that migraine and TTH are closely related conditions that differ mostly in severity.

Interestingly, diabetes showed a strong negative association with migraine (for TTH, a trend in the same direction was observed). This is consistent with findings reported in a large Norwegian population study (Aamodt et al., 2007; Hagen et al., 2017). One hypothesized mechanism is that the vascular changes occurring in diabetes, which cause intimal thickening and stiffness of the blood vessels, might be protective against migraine (Hagen et al., 2017). Regular exercise behavior was also negatively associated with both headache conditions; whether this means that exercising more reduces headache or headache prevents exercise is unclear and requires further study. Furthermore, with respect to the stress variables, it is worth noting that in univariate regression analyses (not shown), financial stress, work stress, and stress at home were all significantly associated with both migraine and TTH. However, in the multivariate analysis, only stress at home remained significant.

Finally, it is worth noting that BMI did not show an association with migraine or TTH in univariate regression analysis (results not shown), while in the multivariate analysis it was associated with both. Possibly this is explained by an age effect: in our data, BMI increased with age, whereas headaches decreased with age. Therefore, when age is not accounted for, the age effect may mask the association between BMI and headache. Extensive investigation of the interactions between the various headache predictors was beyond the scope of this paper, but is worth exploring in more detail in future studies.

This study has limitations. The questionnaire included a detailed assessment of headache symptoms but did not ask patients to report on individual attacks. Therefore, 
patients who experience attacks of both migraine and TTH are most likely to be classified as migraineurs, because TTH is largely defined by the absence of symptoms (e.g., severe headache, pulsating quality, nausea/vomiting, photo- and phono-phobia). Due to the way migraine and TTH are defined in the ICHD-II, it is not possible for an individual to receive both diagnoses based on a single assessment of headache symptomatology, which precludes a diagnosis of TTH once enough migraine symptoms are reported. It is likely that a certain proportion of the individuals classified as migraineurs in this study would also qualify for a diagnosis of TTH if their attacks had been evaluated individually. The mutually exclusive nature of the diagnostic procedure in this design will result in an underestimation of the comorbidity of the two conditions, which may be quite substantial. This is not only an issue in our study, but probably in many studies with similar designs. Traditional bivariate twin models, which are typically used to assess comorbidity and shared (genetic) etiology, are not possible in this design because there is no 'within-person comorbidity' (i.e., one person cannot have both diagnoses). We circumvented this problem by looking at comorbidity within twin pairs. However, our power to detect whether this is due to genetic effects is limited by the likely underestimation of this comorbidity.

In summary, our study showed significant comorbidity between migraine and TTH, and the risk patterns observed were consistent with this comorbidity being party genetic. In addition, migraine and TTH shared many of the same predictors, and typically, the observed effects were in the same direction but stronger for migraine than for TTH, consistent with the view that TTH and migraine represent the mild and severe ends of the same spectrum. These findings suggest that migraine and TTH are etiologically related conditions. This implies that the strict distinction between migraine and TTH as made in the ICHD is at least to some extent artificial, and it would be best to view TTH as a condition etiologically related to migraine, rather than its negation.

\section{Acknowledgments}

We are grateful for the participation of twin families in this research project. Data collection and zygosity typing were made possible by multiple grants from the Netherlands Organization for Scientific Research (NWO): 575-25006, 480-04-004, 904-61-090, 904-61-193, 400-05-717, 311 60008, SPI 56-464-14192 and the Avera Institute for Human Genetics. We gratefully acknowledge Grant NWO 480-15001/674: Netherlands Twin Registry Repository: researching the interplay between genome and environment.

\section{Disclosure of Interests}

None.

\section{References}

Aamodt, A. H., Stovner, L. J., Midthjell, K., Hagen, K., \& Zwart, J. A. (2007). Headache prevalence related to diabetes mellitus. The Head-HUNT study. European Journal of Neurology, 14, 738-744.

Achenbach, T. M., \& Rescorla, L. A. (2003). Manual for the ASEBA adult forms and profiles. Burlington, VT: Research Center for Children, Youth, \& Families, University of Vermont.

Ainsworth, B. E., Haskell, W. L., Whitt, M. C., Irwin, M. L., Swartz, A. M., Strath, S. J., ... Leon, A. S. (2000). Compendium of physical activities: An update of activity codes and MET intensities. Medicine and Science in Sports and Exercise, 32, S498-S504.

Anttila, V., Winsvold, B. S., Gormley, P., Kurth, T., Bettella, F., McMahon, G., ... Palotie, A. (2013). Genome-wide metaanalysis identifies new susceptibility loci for migraine. $\mathrm{Na}$ ture Genetics, 45, 912-917.

Bigal, M. E., \& Lipton, R. B. (2006). Obesity is a risk factor for transformed migraine but not chronic tension-type headache. Neurology, 67, 252-257.

Blumenfeld, A., Schim, J., \& Brower, J. (2010). Pure tensiontype headache versus tension-type headache in the migraineur. Current Pain and Headache Reports, 14, 465-469.

Boomsma, D. I., Busjahn, A., \& Peltonen, L. (2002). Classical twin studies and beyond. Nature Reviews Genetics, 3, 872882.

Breslau, N., Schultz, L. R., Welch, K. M. A., Lipton, R. B., \& Stewart, W. F. (2003). Comorbidity of migraine and depression: Investigating potential etiology and prognosis. $\mathrm{Neu}$ rology, 60, 1308-1312.

Chasman, D. I., Schürks, M., Anttila, V., de Vries, B., Schminke, U., Launer, L. J., ... Kurth, T. (2011). Genomewide association study reveals three susceptibility loci for common migraine in the general population. Nature Genetics, 43, 695-698.

Drummond, P. D., \& Lance, J. (1984). Clinical diagnosis and computer analysis of headache symptoms. Journal of Neurology, Neurosurgery \& Psychiatry, 47, 128-133.

Featherstone, H. J. (1985). Migraine and muscle contraction headaches: A continuum. Headache: The Journal of Head and Face Pain, 25, 194-198.

Freilinger, T., Anttila, V., de Vries, B., Malik, R., Kallela, M., Terwindt, G. M., ... International Headache Genetics Consortium. (2012). Genome-wide association analysis identifies susceptibility loci for migraine without aura. Nature Genetics, 44, 777-782.

Fricton, J., Velly, A., Ouyang, W., \& Look, J. O. (2009). Does exercise therapy improve headache? A systematic review with meta-analysis. Current Pain and Headache Reports, 13, 413-419.

Geels, L. M., Vink, J. M., van Beek, J. H. D. A., Bartels, M., Willemsen, G., \& Boomsma, D. I. (2013). Increases in alcohol consumption in women and elderly groups: Evidence from an epidemiological study. BMC Public Health, 13, 207.

Gormley, P., Anttila, V., Winsvold, B. S., Palta, P., Esko, T., Pers, T. H., ... Palotie, A. (2016). Meta-analysis of 375,000 
individuals identifies 38 susceptibility loci for migraine. $\mathrm{Na}$ ture Genetics, 48, 856-866.

Hagen, K., Åsvold, B. O., Midthjell, K., Stovner, L. J., Zwart, J.-A., \& Linde, M. (2017). Inverse relationship between type 1 diabetes mellitus and migraine. Data from the Nord-Trøndelag Health Surveys 1995-1997 and 20062008. Cephalalgia. Advance online publication.

Haque, B., Rahman, K. M., Hoque, A., Hasan, A. H., Chowdhury, R. N., Khan, S. U., ... Mohammad, Q. D. (2012). Precipitating and relieving factors of migraine versus tension type headache. BMC Neurology, 12, 82 .

Headache Classification Committee of the International Headache Society. (1988). Classification and diagnostic criteria for headache disorders, cranial neuralgias and facial pain. Cephalalgia, 8, 1-96.

Headache Classification Committee of the International Headache Society. (2004). The International Classification of Headache Disorders: 2nd edition. Cephalalgia, 24, 1-160.

Headache Classification Committee of the International Headache Society. (2013). The International Classification of Headache Disorders (beta version). Cephalalgia, 33, 629808.

Hughes, M. E., Waite, L. J., Hawkley, L. C., \& Cacioppo, J. T. (2004). A short scale for measuring loneliness in large surveys results from two population-based studies. Research on Aging, 26, 655-672.

Kaniecki, R. G. (2002). Migraine and tension-type headache an assessment of challenges in diagnosis. Neurology, 58, S15-S20.

Leston, J. (1996). Migraine and tension-type headache are not separate disorders. Cephalalgia, 16, 220-223.

Ligthart, L., Gerrits, M. M., Boomsma, D. I., \& Penninx, B. W. (2013). Anxiety and depression are associated with migraine and pain in general: An investigation of the interrelationships. The Journal of Pain, 14, 363-370.

Ligthart, L., Visscher, C. M., van Houtem, C. M. H. H., Geels, L. M., Vink, J. M., de Jongh, A., ... Boomsma, D. I. (2014). Comorbidity among multiple pain symptoms and anxious depression in a Dutch population sample. The Journal of Pain, 15, 945-955.

Merikangas, K. R., Merikangas, J. R., \& Angst, J. (1993). Headache syndromes and psychiatric disorders: Association and familial transmission. Journal of Psychiatric Research, 27, 197-210.

Mulder, E. J., Van Baal, C., Gaist, D., Kallela, M., Kaprio, J., Svensson, D. A., ... Palotie, A. (2003). Genetic and environmental influences on migraine: A twin study across six countries. Twin Research, 6, 422-431.

Queiroz, L. P., Peres, M. F. P., Piovesan, E. J., Kowacs, F., Ciciarelli, M. C., Souza, J. A., \& Zukerman, E. (2009). A nationwide population-based study of tension-type headache in Brazil. Headache: The Journal of Head and Face Pain, 49, $71-78$.
Rasmussen, B. K. (1993). Migraine and tension-type headache in a general population: Precipitating factors, female hormones, sleep pattern and relation to lifestyle. Pain, 53, 6572.

Rasmussen, B. K. (1996). Migraine and tension-type headache are separate disorders. Cephalalgia, 16, 217-219.

Rasmussen, B. K., Jensen, R., Schroll, M., \& Olesen, J. (1991). Epidemiology of headache in a general population: A prevalence study. Journal of Clinical Epidemiology, 44, 1147-1157.

Russell, M. B., Levi, N., \& Kaprio, J. (2007). Genetics of tension-type headache: A population based twin study. American Journal of Medical Genetics Part B: Neuropsychiatric Genetics, 144, 982-986.

Russell, M. B., Šaltytš-Benth, J., \& Levi, N. (2006). Are infrequent episodic, frequent episodic and chronic tensiontype headache inherited? A population-based study of 11 199 twin pairs. The Journal of Headache and Pain, 7, 119-126.

Scher, A., Terwindt, G., Picavet, H., Verschuren, W., Ferrari, M., \& Launer, L. (2005). Cardiovascular risk factors and migraine The GEM population-based study. Neurology, 64, 614-620.

Schoonman, G. G., Evers, D. J., Ballieux, B. E., de Geus, E. J., de Kloet, E. R., Terwindt, G. M., ... Ferrari, M. D. (2007). Is stress a trigger factor for migraine? Psychoneuroendocrinology, 32, 532-538.

Spierings, E. L. H., Ranke, A. H., \& Honkoop, P. C. (2001). Precipitating and aggravating factors of migraine versus tension-type headache. Headache: The Journal of Head and Face Pain, 41, 554-558.

Stensland, S. Ø., Thoresen, S., Wentzel-Larsen, T., Zwart, J. A., \& Dyb, G. (2014). Recurrent headache and interpersonal violence in adolescence: The roles of psychological distress, loneliness and family cohesion: The HUNT study. The Journal of Headache and Pain, 15, 1-9.

Ulrich, V., Russell, M. B., Jensen, R., \& Olesen, J. (1996). A comparison of tension-type headache in migraineurs and in non-migraineurs: A population-based study. Pain, 67, 501-506.

Vargas, B. B. (2008). Tension-type headache and migraine: Two points on a continuum? Current Pain and Headache Reports, 12, 433-436.

Waters, W. E. (1973). The epidemiological enigma of migraine. International Journal of Epidemiology, 2, 189194.

Willemsen, G., Vink, J. M., Abdellaoui, A., den Braber, A., van Beek, J. H. D. A., Draisma, H. H. M., ... Boomsma, D. I. (2013). The Adult Netherlands Twin Register: 25 years of survey and biological data collection. Twin Research and Human Genetics, 16, 271-281. 